\begin{tabular}{|c|l|}
\hline Title & $\begin{array}{l}\text { Influence of ordered arrangements of cluster chains on the hopping transport in GaA s: MnMnA s hybrids at low } \\
\text { temperatures }\end{array}$ \\
\hline Author(s) & Elm, M. T.; KIar, P. J.; Ito, S.; Hara, S. \\
\hline Citation & $\begin{array}{l}\text { Physical Review B, 83(23), 235305 } \\
\text { https:/doi.org/_0.1103/PhysRevB.83.235305 }\end{array}$ \\
\hline Issue Date & 2011-06-15 \\
\hline Doc URL & http://hdl.handle.net/2115/45815 \\
\hline Rights & O2011 A merican Physical Society \\
\hline Type & article \\
\hline File Information & PRB83_23_235305.pdf \\
\hline
\end{tabular}

Instructions for use 


\title{
Influence of ordered arrangements of cluster chains on the hopping transport in GaAs:Mn/MnAs hybrids at low temperatures
}

\author{
M. T. Elm* and P. J. Klar \\ Institute of Experimental Physics I, Justus-Liebig University, Heinrich-Buff-Ring 16, D-35392 Giessen, Germany \\ S. Ito and S. Hara \\ Research Center for Integrated Quantum Electronics, Hokkaido University, Sapporo 060-8628, Japan \\ (Received 17 February 2011; revised manuscript received 24 March 2011; published 6 June 2011)
}

\begin{abstract}
Two different arrangements of cluster chains consisting of elongated MnAs nanoclusters were deposited on (111)B-GaAs substrates by selective-area metal-organic vapor-phase epitaxy. This method allows one a controlled positioning of the nanoclusters on the substrate, offering the possibility to investigate the influence of the nanocluster arrangement on the transport properties of granular hybrid structures. Magnetotransport measurements at low temperatures were performed for both cluster-chain arrangements prepared. In contrast to GaAs:Mn/MnAs hybrids with a random cluster distribution, which usually show a negative magnetoresistance at low temperatures, large positive magnetoresistance effects are observed for cluster-chain arrangements at $15 \mathrm{~K}$. Furthermore, the arrangements exhibit a rectifying behavior of the current-voltage characteristics in the presence of an external magnetic field. The magnitude of both effects correlates with the arrangement of the chains on the substrate and can be explained qualitatively by assuming hopping conduction as the dominant transport mechanism.
\end{abstract}

DOI: 10.1103/PhysRevB.83.235305

PACS number(s): 72.20.Ee, 75.50.Pp, 81.16.-c, 72.20.My

\section{INTRODUCTION}

Semiconductor-ferromagnetic granular hybrid structures, consisting of ferromagnetic nanoclusters embedded into a semiconducting host matrix, gained much attention in recent years, because they show a strong correlation between transport and magnetic properties ${ }^{1,2}$ as well as magnetoresistance (MR) effects similar to giant magnetoresistance (GMR) or the tunneling magnetoresistance (TMR) ${ }^{3-5}$ Moreover, the large number of degrees of freedom in such a composite material results in a huge tunability of its properties compared with a single-phase material, making the hybrids a promising material system for new magnetoelectronic devices. For example, the cluster size strongly contributes to the hybrids' properties. ${ }^{6,7}$

Conventionally synthesized GaAs:Mn/MnAs hybrid structures usually exhibit a random cluster distribution in the host matrix, which leads to significant variations in the transport behavior. The random cluster distribution hinders a utilization of the hybrids in miniaturized magnetoelectronic devices, e.g., a reproducibility of the hybrids' properties cannot be achieved. Technological applications therefore are mainly restricted to macroscopic devices, where the mean distance between adjacent clusters is much smaller than the characteristic size of the device. However, the problem of randomness can be overcome by the method of selective-area growth of self-assembled MnAs nanoclusters on prepatterned (111)B-GaAs subtrates. As shown by Wakatsuki et al. ${ }^{8}$ and Ito et al. ${ }^{9}$ this growth process offers the possibility to control the size, shape, and the position of the nanoclusters on the substrate. This allows one to actively tune the transport behavior of the hybrids by varying the clusters' properties. Especially the cluster arrangement strongly influences the transport paths of the carriers through the host matrix and thus the magnitude of the occuring MR effects. ${ }^{10,11}$ The investigation of the transport properties of hybrids with different cluster arrangements is therefore essential in order to assess the potential of these structures for device applications and to understand the physical background of the clusters' influence on the transport properties.

In this paper, we present the results of magnetotransport measurements at low temperatures of various ordered arrangements of cluster chains consisting of elongated MnAs nanoclusters grown by selective-area metal-organic vapor-phase epitaxy (SA-MOVPE). Usually GaAs:Mn/MnAs hybrids with a random cluster distribution exhibit a negative MR at low temperatures, which can be explained by trapping of the carriers in the vicinity of the nanoclusters. ${ }^{12-15}$ However, for the samples grown by SA-MOVPE a large positive MR effect occurs, which arises due to hopping transport. Similar positive magnetoresistance effects can also be observed in other material systems at low temperatures, where variable range hopping is the dominant transport mechanism. ${ }^{16-18}$

\section{EXPERIMENTAL DETAILS}

Two ordered arrangements of cluster chains consisting of elongated nanoclusters were grown by SA-MOVPE on prepatterned semi-insulating (111)B-GaAs substrates. The (111) orientation of the substrate was chosen because the $c$ plane of the NiAs-type MnAs nanoclusters matches well the (111) plane as both are of the same symmetry. The substrates were masked with $\mathrm{a} \mathrm{SiO}_{2}$ thin film deposited by plasma sputtering. The initial mask openings for a controlled positioning of the nanoclusters were prepared by electron-beam lithography and wet-chemical etching with buffered hydrofluoric acid. The inital mask openings had a width of $150 \mathrm{~nm}$ and a length of $650 \mathrm{~nm}$. For the growth of the cluster chains, the distance between the openings was reduced to $50 \mathrm{~nm}$ to achieve a merging of the clusters during the growth process. Finally, an AlGaAs buffer layer was grown followed by the growth of the MnAs nanoclusters in the initial openings. Further details of the prepatterning and the subsequent growth procedure of 
the buffer layer and the MnAs nanoclusters can be found elsewhere..$^{8,9,11}$ The resulting clusters have a typical length and width of 690 and $290 \mathrm{~nm}$, respectively. During the growth of the nanoclusters, Mn diffuses into the semi-insulating GaAs substrate at the initial openings, where it is incorporated as an acceptor, yielding a p-type paramagnetic diluted magnetic semiconductor with a nonrandom distribution of $\mathrm{Mn}$.

The magnetotransport measurements as well as the measurements of the current-voltage characteristics were carried out at $15 \mathrm{~K}$ using a superconducting magnet system generating external magnetic fields up to $10 \mathrm{~T}$. The external magnetic field was applied perpendicular to the sample surface. DC currents applied along the [11 $\overline{2}$ ]-direction were used, varying between $500 \mathrm{pA}$ and $2 \mathrm{nA}$.

\section{RESULTS AND DISCUSSION}

The cluster chains were investigated by scanning electron microscopy (SEM) using a Hitachi S-4100 electron mircoscope. The corresponding SEM images of the two arrangements with cluster chains prepared are shown in Fig. 1. For the cluster chains of the first arrangement, shown in Fig. 1(a), the single elongated nanoclusters are oriented along the [101] direction of the substrate. In the following, this arrangement is called cluster chains I. The second cluster-chain arrangement, which is shown in Fig. 1(b), consists of an alternating sequence of elongated nanoclusters oriented along

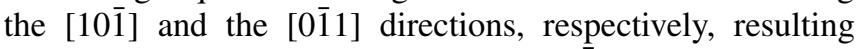
in cluster chains oriented along the [011] direction. This arrangement is referred to as cluster chains II in the following. Both arrangements consist of elongated MnAs nanoclusters of high crystal quality. However, a full merging of the elongated clusters was achieved for the cluster chains I only, where the

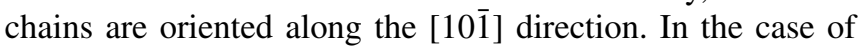
cluster chains II, the chains exhibit small gaps between the single nanoclusters.

Figure 2 shows the temperature dependence of the resistivity of both cluster-chain arrangements investigated as well as for an additional sample with a random cluster distribution. The latter was grown on an undoped GaInAs layer on (111)BInP substrate by standard MOVPE, i.e., without prepatterning of the substrate prior to the cluster growth. A detailed description of the sample growth can be found elsewhere. ${ }^{19}$ For a better comparison, the resistivity of the sample with the random cluster distribution was multiplied by a factor of 50 . At higher temperatures, i.e., for $T>20 \mathrm{~K}$, where traditional band transport is the dominant transport mechanism, a strong non-Arrhenius temperature dependence of the resistivity can

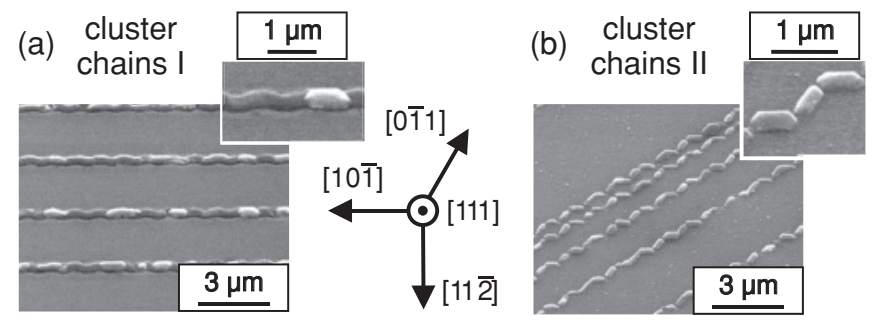

FIG. 1. Scanning electron microscopy images of the two clusterchain arrangements prepared.

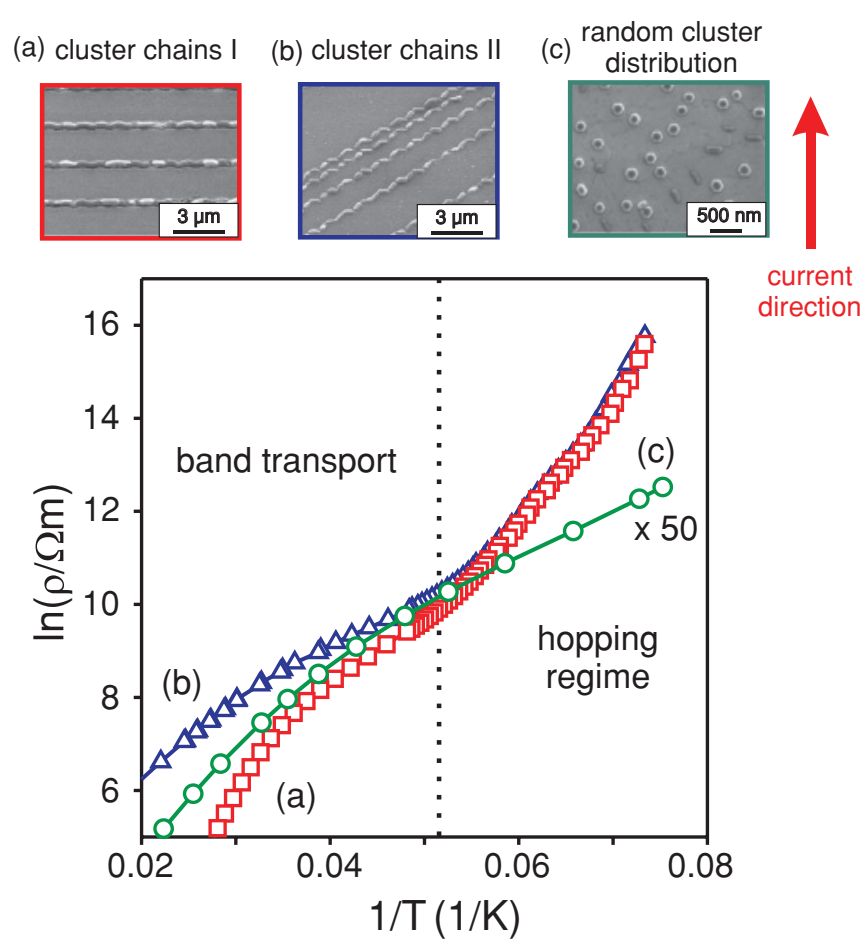

FIG. 2. (Color online) Temperature dependence of the resistivity $\rho$ of the two arrangements investigated with cluster chains I and II as well as of a hybrid structure with a random cluster distribution. Below $20 \mathrm{~K}$, the resisitivity of the two arrangements strongly increases, indicating a change of the dominant transport mechanism from traditional band transport to hopping conductivity.

be observed for all three samples. This behavior indicates an energetically broadened distribution of the acceptor levels at high Mn doping concentrations. As Michel et al. have shown, ${ }^{20}$ such a non-delta-like distribution of acceptor energies leads to a bowing in the Arrhenius plot of the resistivity. Below $20 \mathrm{~K}$, the temperature dependence changes significantly only for the samples with chain-like cluster arrangements. Their resistivity increases almost exponentially with decreasing temperature, while for the sample with a random cluster distribution no change of the behavior can be observed.

To investigate the transport properties of the samples with cluster chains in more detail, magnetic-field-dependent measurements of the resistivity were performed. Figure 3 shows the MR behavior at $15 \mathrm{~K}$ for both cluster-chain arrangements investigated. The MR is defined as $\mathrm{MR}=\left(\rho(H)-\rho_{0}\right) / \rho_{0}$, where $\rho_{0}$ and $\rho(H)$ are the resitivity in zero field and at an applied magnetic field $H$, respectively. Both arrangements show a positive MR effect at this temperature. For the arrangement with cluster chains I a huge MR effect can be observed, which increases up to approximately $3.5 \mathrm{~T}$ to a value of nearly $400 \%$. At $3.5 \mathrm{~T}$ an additional negative MR contribution occurs, which changes the slope of the MR curve. However, the positive MR dominates in the entire magnetic field range investigated and shows a further increase with increasing magnetic field, reaching a value of about $900 \%$ at $\mu_{0} H=10 \mathrm{~T}$. A similar bending of the slope of the magnetoresistance curve can also be observed for the sample with cluster chains II. For this arrangement the MR reaches a value of only about $70 \%$ at $3 \mathrm{~T}$ and remains nearly constant 


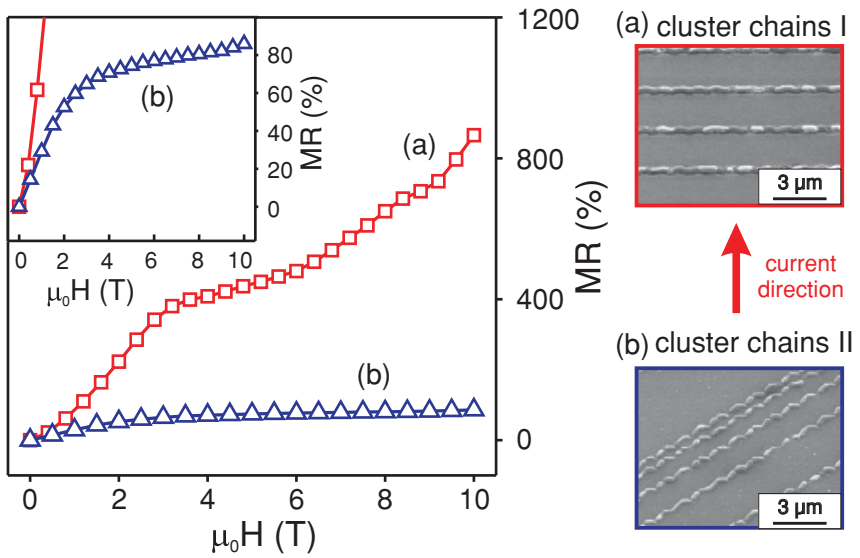

FIG. 3. (Color online) Magnetoresistance of the two cluster arrangements with cluster chains I and II measured at $15 \mathrm{~K}$. The applied current was $500 \mathrm{pA}$. The inset shows a zoom-in of the magnetoresistance for the cluster chains II oriented along the [011] direction

with further increasing fields. Only a small increase up to $85 \%$ at $10 \mathrm{~T}$ can be observed. The large positive MR effects at low temperatures cannot be observed in comparable samples with random cluster distributions. These usually show a dominant negative MR effect. ${ }^{4,15,21,22}$

The strong differences in the transport behavior are caused by different Mn distributions in the matrix, where the transport takes place. In the case of the cluster-chain arrangements, the prepatterning of the sample surface with a $\mathrm{SiO}_{2}$ mask inhibits a diffusion of $\mathrm{Mn}$ into the matrix, which is responsible for its p-type conductivity. Only at the initial openings, where the clusters grow during the MOVPE process, Mn can diffuse into the GaAs substrate. The arrangements with nanocluster chains therefore possess a strongly inhomogeneous Mn distribution in the matrix. In the vicinity of the MnAs nanoclusters the matrix is highly Mn doped, leading to channels of high doping along the cluster chains. The regions between the cluster chains exhibit only a very low Mn concentration. The distribution of the Mn content in the matrix is schematically shown in Fig. 4(a). The much lower Mn concentration in the matrix and its inhomogeneous distribution result in a much higher resistivity compared with those of samples with a random cluster distribution. At low temperatures most of the carriers are not activated into extended band states, leading to hopping between the free acceptor states as the dominant transport mechanism in the matrix. In particular, in the region of the matrix with a low Mn content, hopping takes place between occupied and empty acceptor states. The latter originate from compensation caused by $\mathrm{Mn}$ interstitials, which act as double donors. $^{23}$

The unusual increase of the resistivity can be explained by considering the influence of the nanoclusters' magnetization on the acceptor sites. In the vicinity of the cluster chains, where the Mn content is high, the inhomogeneous stray field of the ferromagnetic nanoclusters causes a local splitting of the acceptor states due to the giant Zeeman splitting (GZS), ${ }^{24,25}$ as schematically shown in Fig. 4(b). Due to this splitting most of the carriers are trapped at the matrix-cluster interface. More impurity sites get occupied, reducing the probability
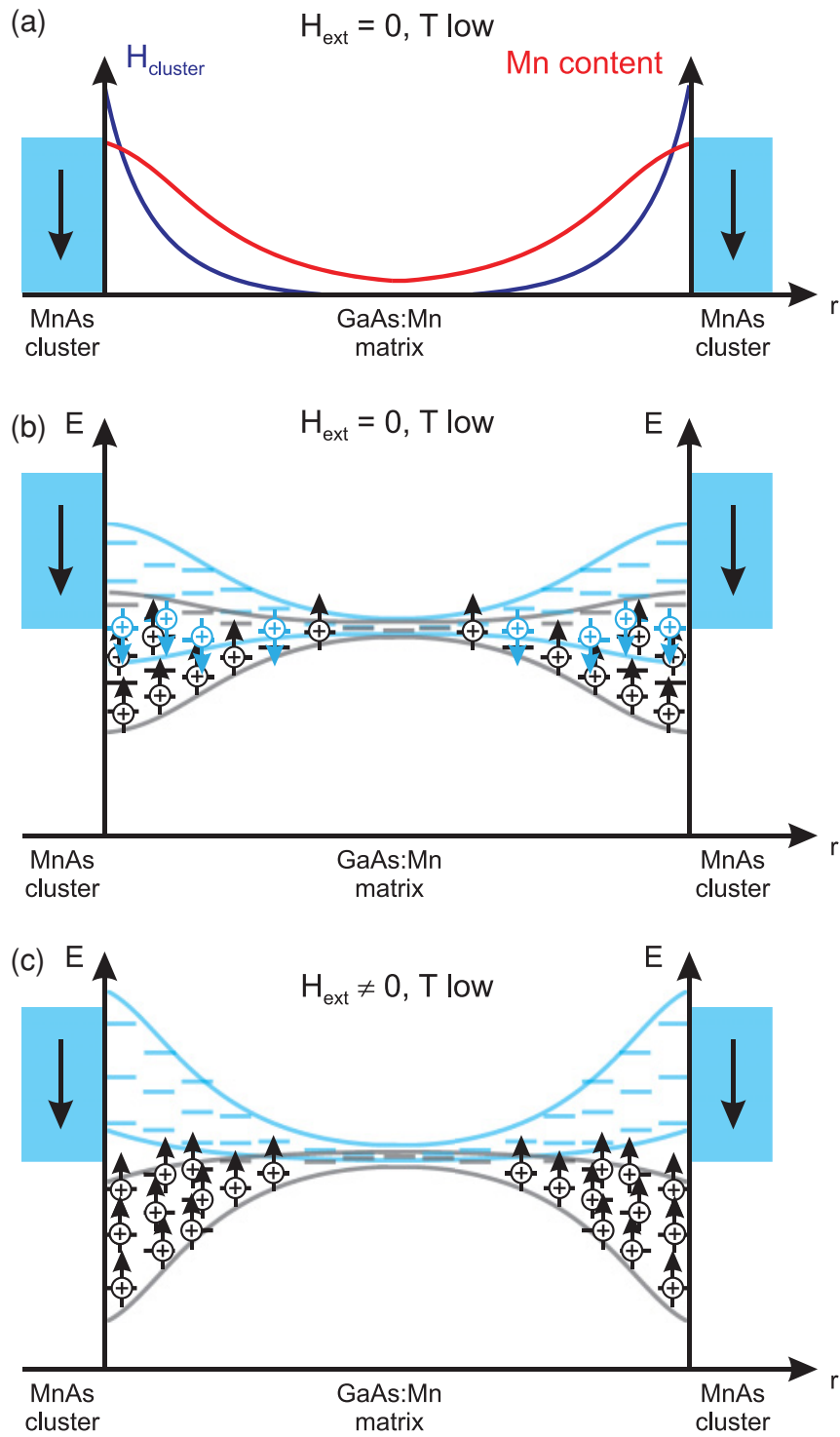

FIG. 4. (Color online) Schematic illustration of the influence of an external magnetic field on the distribution of acceptor states in the matrix. (a) Distribution of the Mn content in the matrix as well as strength of the local magnetic field caused by the ferromagnetic nanoclusters. (b) In the cluster vicinity the local field of the clusters causes a GZS of the acceptor states with different spin orientations. (c) In the presence of an external magnetic field the increasing GZS reduces the overlap of the impurity bands, resulting in a positive MR effect.

of hopping in the cluster vicinity. Furthermore, the number of carriers which can contribute to the transport through the matrix is reduced. With decreasing temperature the GZS increases, leading to the observed exponential increase of the resistivity.

In the case of samples with a random cluster distribution, where no prepatterning step of the surface was carried out, Mn can diffuse into the matrix on the entire sample surface. This results in a random, but almost homogeneous $\mathrm{Mn}$ distribution as well as a higher Mn concentration in the matrix, leading to broadened impurity bands. As a consequence, the temperature dependence of this sample shows regular 
band transport even at low temperatures and a much lower resistance compared with that of the samples with cluster-chain arrangements. In the framework of extended band transport, the negative MR effect for GaAs:Mn/MnAs hybrids with randomly distributed nanoclusters can be explained by a release of the carriers trapped at the cluster-matrix interface. Due to the homogeneous Mn content in the matrix the GZS leads to splitting of the valence bands in the entire matrix. An external magnetic field therefore reduces the energetic depth of the carrier traps at the interface. This results in a release of the carriers and therefore in a negative MR effect. ${ }^{4,11,13,15} \mathrm{~A}$ positive MR occurs only at higher temperatures, which is also in good agreement with theoretical modeling of the transport properties. $^{10}$

The strong positive MR effects observed at $15 \mathrm{~K}$ supports the assumption of hopping conductivity at low temperatures and can be explained with a transport mechanism motivated by Kamimura ${ }^{26}$ and Kamimura et al., ${ }^{27}$ who discussed spin effects in the hopping regime.

In the hopping regime transport is carried by hopping between single impurity sites. The Pauli principle allows, each site to be occupied by only two carriers of different spin orientations. Because of the energetically broadened distribution of the acceptor states, the two impurity subbands with different spin orientations overlap in the absence of an external magnetic field, as schematically shown in Fig. 4(b). Only close to the ferromagnetic nanoclusters the local GZS may result in a splitting of both spin subbands, reducing the overlap of the impurity bands. However, near the nanoclusters, where both impurity subbands overlap, hopping can take place between impurity sites of both spin orientations, i.e., hopping can occur between doubly occupied to single occupied or empty states as well as between singly occupied ones.

In the presence of a magnetic field the GZS also causes a splitting of the impurity levels in the entire matrix. Due to the inhomogeneous Mn distribution, this splitting is largest near the nanoclusters, where the Mn content is high, and lowest in the regions away from the cluster chains. The increasing splitting in the cluster vicinity reduces the overlap between the two impurity spin subbands, as schematically shown in Fig. 4(c), i.e., due to the external magnetic field only carriers of one spin orientation can be found in this region. Additionally, the region of the matrix, where the impurity bands still overlap, extends further away from the clusters into regions where the number of acceptor states is low. Thus, applying a magnetic field drastically reduces the number of possible hopping sites leading to a positive MR effect.

Also the negative MR contribution arising at about $3 \mathrm{~T}$ can be explained by assuming hopping conductivity at low temperatures. The GZS not only leads to a shift of the broadened impurity subbands, but also results in a change of their energetic width. Because the GZS depends linearly on the effective Mn concentration, the energy splitting is larger for higher Mn concentration. In the matrix, where the hopping takes place, the higher-energy acceptor states approach those of lower energy, resulting in a shrinkage of the energetic bandwidth. The shrinking width of the impurity band increases the number of possible hopping sites near the Fermi energy, yielding a negative contribution to the MR.

The assumptions made above in order to describe the behavior of the MR qualitatively cannot explain the strong difference in the magnitude of the effects for the two different cluster arrangements. As shown by Michel et al. in the regime of extended band transport the magnitude of the positive MR in the intermediate temperature range is strongly determined by the cluster arrangement. ${ }^{10}$ This means that, also in the hopping regime, an additional positive contribution to the MR arises from the distribution of the nanoclusters in the matrix. The basic idea to explain this additional MR contribution is based on the assumption that the paramagnetic matrix and the half-metallic MnAs clusters possess carriers with different majority and minority spins. A schematic illustration of the proposed mechanism is shown in Fig. 5. In the absence of a magnetic field the hopping conductivity is carried by holes from both impurity spin subbands near the Fermi energy. However, only one spin orientation of the carriers can pass preferably through the nanoclusters due to the ferromagnetic clusters' spin polarization at the Fermi energy, as schematically shown in Fig. 5(a).
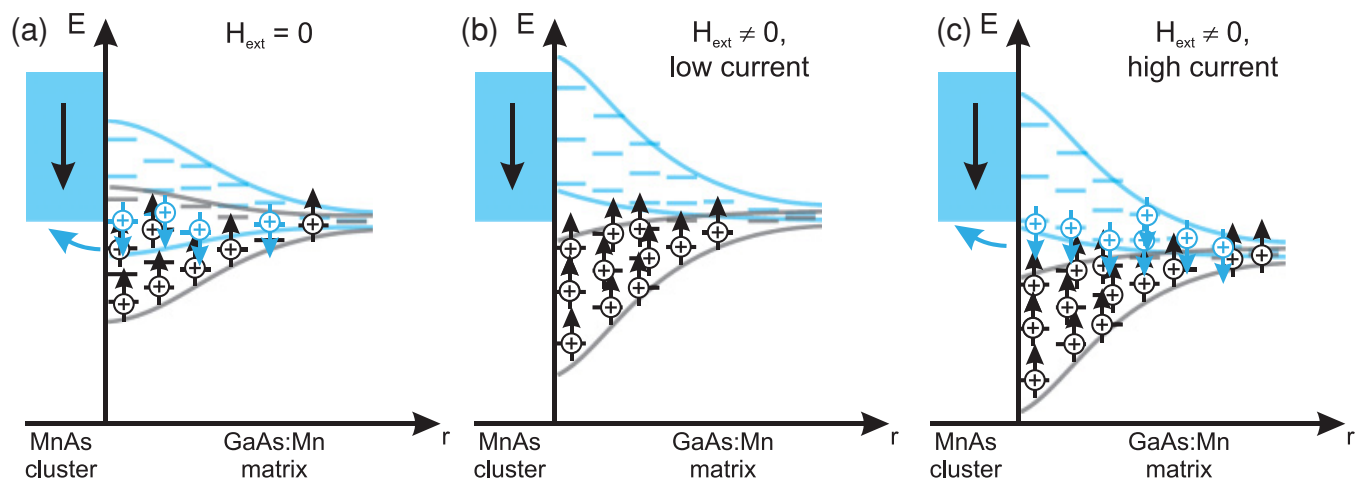

FIG. 5. (Color online) Schematic illustration of the additional positive contribution to the MR caused by the cluster arrangements. Due to compensation the number of holes is smaller than the number of corresponding acceptor states. (a) In the absence of an external magnetic field, carriers with a spin orientation parallel to the clusters' polarization can pass the nanocluster. (b) If a magnetic field is applied, only carriers with a spin orientation opposite to the clusters' polarization carry the transport. The transport through the clusters is therefore suppressed. (c) Increasing the applied current increases also the number of minority carriers in the impurity bands, which can pass the clusters, and the MR effect is reduced. 
In the presence of an external field shown in Fig. 5(b) the splitting of the impurity bands leads to an alignment of the carrier spins in the matrix. Thus, hopping transport is carried by only one spin orientation of the carriers in the matrix. Because the carriers with majority spin of the matrix and the nanoclusters exhibit opposite spin orientations, the carriers of the matrix cannot pass the nanoclusters anymore, resulting in an additional positive MR contribution. A similar behavior can also be found in the regime of extended band transport at higher temperatures. ${ }^{4,10,13}$ In the case of the arrangement with cluster chains I, where the chains are arranged perpendicular to the current direction, the clusters suppress the transport through the matrix. The current cannot find a path through the matrix without having to pass a nanocluster yielding a large positive contribution to the MR. Because for the second arrangement the cluster chains are oriented along the [011] direction, i.e., nearly parallel to the applied current direction, there always exists a current path through the entire GaAs:Mn matrix parallel to the cluster chains. The influence of the nanoclusters on the transport is therefore almost negligible; thus a much smaller positive MR is observed.

Besides the large MR effects, the arrangements with cluster chains investigated also show a strong dependence of the magnitude of the MR on the applied current, as shown in Fig. 6(a). For both arrangements, the magnitude of the positive MR decreases with increasing current, while the qualitative behavior remains unaffected. For the arrangement with cluster chains I, the MR effect at $\mu_{0} H=10 \mathrm{~T}$ is drastically reduced
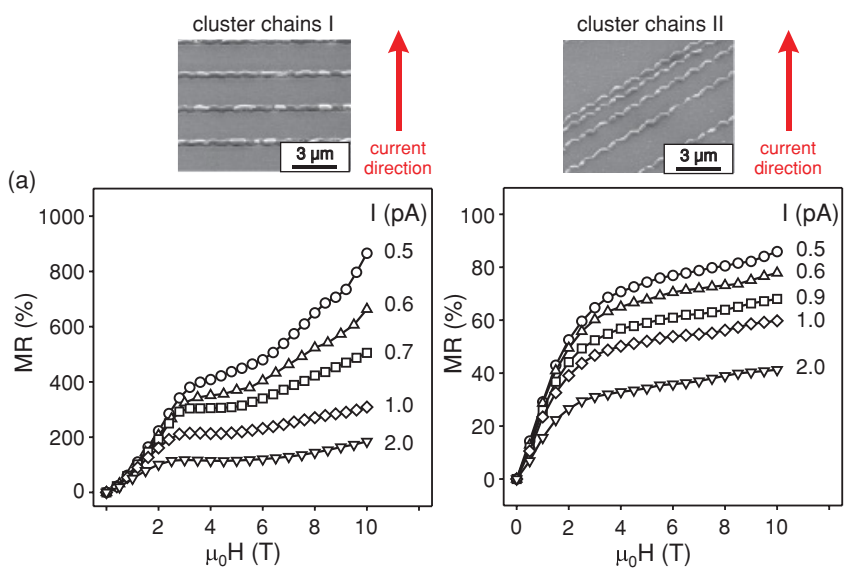

(b)
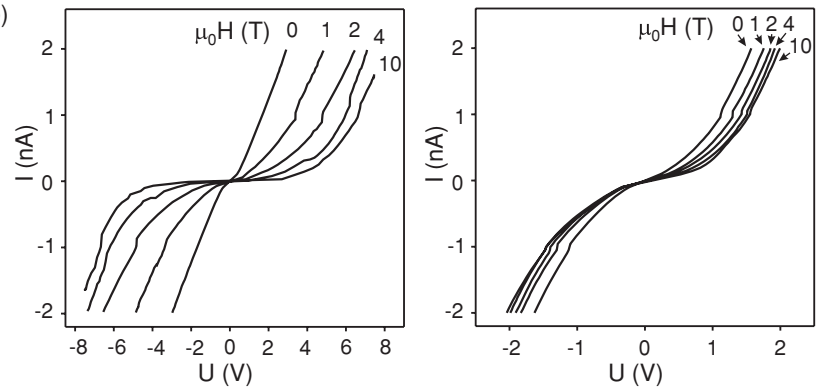

FIG. 6. (Color online) (a) Dependence of the MR effects for the arrangement with cluster chains I and with cluster chains II. Increasing the applied current strongly reduces the positive MR effects. (b) Corresponding current-voltage characteristics of both arrangements for different external magnetic fields. The measurements were performed at $15 \mathrm{~K}$. from nearly $900 \%$ to about only $180 \%$, if the applied current is increased from $500 \mathrm{pA}$ to $2 \mathrm{nA}$. For the arrangement with cluster chains II, the effect is less pronounced. The MR decreases from only $85 \%$ to about $42 \%$ in the same current range.

To investigate this behavior in more detail, measurements of the current-voltage characteristics of both types of cluster chains were carried out for different applied magnetic fields at $15 \mathrm{~K}$. The results are shown in Fig. 6(b). Both clusterchain arrangements show nearly an ohmic behavior in the absence of a magnetic field. The weak rectifying behavior is probably due to the formation of a Schottky barrier at the cluster-matrix interface. ${ }^{14,28}$ For the arrangement of cluster chains I, the rectifying behavior becomes strongly pronounced with increasing magnetic field. This leads to the observed dependence of the magnitude of the MR on the applied current described above. For the arrangement with cluster chains II only a small rectifying behavior can be observed in the presence of an external magnetic field. At temperatures above $30 \mathrm{~K}$, where transport in extended band states is dominant, both cluster arrangements exhibit an ohmic behavior of the current-voltage characteristics (not shown here). Because both arrangements show an almost ohmic behavior in the absence of an external magnetic field, heating effects due to the applied current can be excluded as origin for the rectifying behavior observed at $15 \mathrm{~K}$.

The rectifying behavior of the current-voltage characteristics in an external magnetic field can also be explained considering the influence of the nanocluster arrangements on the hopping transport. As discussed above, in the presence of a magnetic field the carrier transport through the ferromagnetic nanocluster is suppressed. However, increasing the current applied to the cluster-chain arrangement, more carriers are injected into the GaAs:Mn matrix. As a consequence the number of carriers in the impurity bands increases, as schematically shown in Fig. 5(c), and the Fermi energy is shifted to higher energies. Therefore also impurity sites in the second spin subband will be occupied, increasing the number of carriers with minority spins in the matrix. In contrast to the carriers with majority spins, the carriers with minority spins possess the same spin orientation as the ferromagnetic nanoclusters. Therefore they can pass the clusters even in a magnetic field, yielding a reduction of the positive MR effect.

The assumption that the nanoclusters are responsible for the rectifying behavior also explains the strong differences in the magnetic field dependence of the current-voltage characteristic of the two arrangements investigated. Because the cluster chains I strongly influence the transport through the matrix due to their orientation perpendicular to the current direction, this arrangement shows a large increase of the rectifying behavior in a magnetic field. For the arrangement with cluster chains II, where the transport through the matrix is nearly unaffected by the cluster chains, only a weak influence of the magnetic field on the current-voltage characteristics is observed.

\section{SUMMARY}

Two different cluster-chain arrangements grown by SAMOVPE on prepatterned substrates were investigated by magnetotransport measurements at a temperature of $15 \mathrm{~K}$. 
During the growth process Mn can diffuse into the semiinsulating substrate only at the initial openings of the $\mathrm{SiO}_{2}$ mask, leading to a lower Mn concentration in the matrix compared with hybrids grown by standard MOVPE. As a consequence, at low temperatures the dominating transport mechanism is determined by hopping conductivity. The two arrangements exhibit large positive MR effects as well as a rectifying current-voltage characteristics, whose magnitudes are strongly correlated with the cluster arrangements. Both effects can be qualitatively understood in the hopping regime, if the influence of the nanocluster arrangement on the transport path through the matrix is considered. The large dependence of the MR effects on the cluster-chain arrangement offers the possibility to actively tune the hybrids' properties for studying hopping transport in dilute magnetic semiconductor hybrid structures.

\section{ACKNOWLEDGMENTS}

We would like to thank the DFG and JSPS for financial support. M.T.E. would like to thank the Justus-Liebig University for providing his $\mathrm{PhD}$ scholarship. *matthias.elm@physik.uni-giessen.de

${ }^{1}$ S. A. Wolf, D. D. Awschalom, R. A. Buhrman, J. M. Daughton, S. von Molnár, M. L. Roukes, A. Y. Chtchelkanova, and D. M. Treger, Science 294, 1488 (2001).

${ }^{2}$ H. Ohno, J. Magn. Magn. Mater. 200, 110 (1999).

${ }^{3}$ A. E. Berkowitz, J. R. Mitchell, M. J. Carey, A. P. Young, S. Zhang, F. E. Spada, F. T. Parker, A. Hutten, and G. Thomas, Phys. Rev. Lett. 68, 3745 (1992).

${ }^{4}$ W. Heimbrodt, P. J. Klar, S. Ye, M. Lampalzer, C. Michel, S. D. Baranovskii, P. Thomas, and W. Stolz, J. Supercond. 18, 315 (2005).

${ }^{5}$ J. Q. Xiao, J. S. Jiang, and C. L. Chien, Phys. Rev. Lett. 68, 3749 (1992).

${ }^{6}$ H.-A. Krug von Nidda, T. Kurz, A. Loidl, T. Hartmann, P. J. Klar, W. Heimbrodt, M. Lampalzer, K. Volz, and W. Stolz, J. Phys. Condens. Matter 18, 6071 (2006).

${ }^{7}$ C. Brosseau, J. B. Youssef, P. Talbot, and A.-M. Kohn, J. Appl. Phys. 93, 9243 (2007).

${ }^{8}$ T. Wakatsuki, S. Hara, S. Ito, D. Kawamura, and T. Fukui, Jpn. J. Appl. Phys. 48, 04C137 (2009).

${ }^{9}$ S. Ito, S. Hara, T. Wakatsuki, and T. Fukui, Appl. Phys. Lett. 94, 243117 (2009).

${ }^{10}$ C. Michel, M. T. Elm, B. Goldlücke, S. D. Baranovskii, P. Thomas, W. Heimbrodt, and P. J. Klar, Appl. Phys. Lett. 92, 223119 (2008).

${ }^{11}$ M. T. Elm, C. Michel, J. Stehr, D. M. Hofmann, P. J. Klar, S. Ito, S. Hara, and H.-A. Krug von Nidda, J. Appl. Phys. 107, 013701 (2010).

${ }^{12}$ K. Ganesan and H. L. Bhat, J. Appl. Phys. 103, 043701 (2008).

${ }^{13}$ W. Heimbrodt and P. J. Klar, Local-Moment Ferromagnets, edited by M. Donath and W. Nolting, Lecture Notes in Physics (Springer, Berlin/Heidelberg 2005), Vol. 678, p. 167.
${ }^{14}$ C. Michel, C. H. Thien, S. Ye, P. J. Klar, W. Heimbrodt, S. D. Baranovskii, P. Thomas, M. Lampalzer, K. Volz, W. Stolz, and B. Goldlücke, Superlattices Microstruct. 37, 321 (2005).

${ }^{15}$ S. Ye, P. J. Klar, Th. Hartmann, W. Heimbrodt, M. Lampalzer, S. Nau, T. Torunski, W. Stolz, T. Kurz, H.-A. Krug von Nidda, and A. Loidl, Appl. Phys. Lett. 83, 3927 (2003).

${ }^{16}$ K. G. Lisunov, E. K. Arushanov, Ch. Kloc, J. Broto, J. Leotin, H. Rokoto, M. Respaud, and E. Bucher, Phys. B 229, 37 (1996).

${ }^{17}$ L.-J. Chen, D.-Y. Wang, Q.-F. Zhan, W. He, and Q.-A. Li, Chin. Phys. Lett. 25, 2625 (2008).

${ }^{18}$ J. Stankiewicz, Phys. Rev. B 42, 10597 (1990).

${ }^{19}$ S. Hara, D. Kawamura, H. Iguchi, J. Motohisa, and T. Fukui, J. Cryst. Growth 310, 2390 (2008).

${ }^{20}$ C. Michel, S. D. Baranovskii, P. J. Klar, P. Thomas, B. Goldlücke, Appl. Phys. Lett. 89, 112116 (2006).

${ }^{21}$ H. Akinga, J. De Boeck, G. Borghs, S. Miyanishi, A. Asamitsu, W. Van Roy, Y. Tomioka, and L. H. Kuo, Appl. Phys. Lett. 72, 3368 (1998).

${ }^{22}$ P. J. Wellmann, J. M. Garcia, J.-L. Feng, and P. M. Petroff, Appl. Phys. Lett. 73, 3291 (1998).

${ }^{23}$ F. Máca and J. Mašek, Phys. Rev. B 65, 235209 (2002).

${ }^{24}$ W. Heimbrodt, Th. Hartmann, P. J. Klar, M. Lampalzer, W. Stolz, K. Volz, A. Schaper, W. Treutmann, H.-A. Krug von Nidda, A. Loidl, T. Ruf, and V. F. Sapega, Phys. E 10, 175 (2001).

${ }^{25}$ J. K. Furdyna, J. Appl. Phys. 64, R29 (1988).

${ }^{26}$ H. Kamimura, Philos. Mag. Part B 52, 541 (1985).

${ }^{27}$ H. Kamimura, A. Kurobe, and T. Takemori, Phys. B + C 117, 652 (1983).

${ }^{28}$ P. B. Parchinskiy, F. C. Yu, C. Gao, S. W. Lee, D. Kim, H. Kim, and Y. E. Ihm, J. Electroceram. 17, 1047 (2006). 